\title{
Hadron Phenomenology from Quenched Overlap Fermions
}

David Galletly ${ }^{\mathrm{a}}$, Martin Gürtler ${ }^{\mathrm{b}}$, Roger Horsley ${ }^{\mathrm{a}}$, Karl Koller ${ }^{\mathrm{c}}$, Volkard Linke ${ }^{\mathrm{d}}$, Paul E.L. Rakow ${ }^{\mathrm{e}}$, Charles J. Roberts ${ }^{\mathrm{e}}$, Gerrit Schierholz ${ }^{\mathrm{b}}$, Thomas Streuer ${ }^{\mathrm{b} *}$

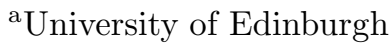

Edinburgh EH9 3JZ, UK

${ }^{\mathrm{b}}$ John von Neumann-Institut für Computing NIC

Platanenalleee 6, 15738 Zeuthen, Germany

'Sektion Physik, Universität München

80333 München, Germany

${ }^{\mathrm{d}}$ Institut für Theoretische Physik, Freie Universität Berlin

14196 Berlin, Germany

e Theoretical Physics Division, Department of Mathematical Sciences, University of Liverpool Liverpool L69 3BX, UK

We compute hadron masses and nucleon matrix elements in quenched QCD using overlap fermions.

\section{INTRODUCTION}

Hadron properties at small quark mass depend strongly on the chiral symmetries of QCD. Therefore, when doing lattice calculations, it is advantageous to use an action which respects chiral symmetry, as provided by the overlap fermion action.

\section{LATTICE SIMULATION}

We use the overlap operator given by

$D=\rho\left(1+\frac{m_{q}}{2 \rho}+\left(1-\frac{m_{q}}{2 \rho}\right) \gamma_{5} \operatorname{sgn}\left(H_{W}(-\rho)\right)\right)$,

where $H_{W}(-\rho)=\gamma_{5}\left(D_{W}-\rho\right), D_{W}$ being the Wilson Dirac operator. We approximate the sign function appearing in (1) by minmax polynomials [2]. For the gauge part we chose the Lüscher-

*Speaker
Weisz action $[3]$

$$
\begin{aligned}
S[U] & =\frac{6}{g^{2}}\left(c_{0} \sum_{\text {plaq }} \frac{1}{3} \operatorname{Re} \operatorname{Tr}\left[1-U_{\text {plaq }}\right]\right. \\
& +c_{1} \sum_{\text {rect }} \frac{1}{3} \operatorname{Re} \operatorname{Tr}\left[1-U_{\text {rect }}\right] \\
& \left.+c_{2} \sum_{\text {par }} \frac{1}{3} \operatorname{Re} \operatorname{Tr}\left[1-U_{\text {par }}\right]\right),
\end{aligned}
$$

with coefficients $c_{1}, c_{2}\left(c_{0}+8 c_{1}+8 c_{2}=1\right)$ taken from tadpole improved perturbation theory [4]. We ran our computations at three lattice spacings, see table 1 . The scale has been set from the pion decay constant. The parameter $\rho$ in (1) was

Table 1

\begin{tabular}{|c|c|c|c|}
\hline$V$ & $\beta$ & $a(\mathrm{fm})$ & confs. \\
\hline $12^{3} 24$ & 7.60 & $0.229(4) \mathrm{fm}$ & 200 \\
$16^{3} 32$ & 8.00 & $0.153(3) \mathrm{fm}$ & 300 \\
$24^{3} 48$ & 8.45 & $0.105(2) \mathrm{fm}$ & 200 \\
\hline
\end{tabular}

Parameters of the gauge configurations used. 
set to 1.4 .

In order to remove $O(a)$ errors from matrix elements, we employ the method of [5], which amounts to replacing propagators $D^{-1} \Psi$ by $\frac{1}{1-\frac{m}{2 \rho}} D^{-1} \Psi-\frac{1}{2\left(1-\frac{m}{2 \rho}\right)} \Psi$. Jacobi smeared point sources [1] with parameters $N_{s}=50$ and $\kappa_{s}=0.21$ have been used in order to obtain a good overlap with the ground state.

\section{HADRON MASSES}

Quenched chiral perurbation theory predicts the following dependence of the pion mass on the quark mass:

$m_{\pi}^{2}=A m_{q}\left(1-\delta\left(1+\log \frac{A m_{q}}{\Lambda_{\chi}^{2}}\right)\right)+b m_{q}^{2}$,

with

$A=\frac{2 \Sigma}{f^{2}}$,

where $\Sigma$ and $f$ are coupling constants in the chiral effective Lagrangian which in leading order correspond to the chiral condensate and the pion decay constant, and with

$\delta=\frac{m_{0}^{2}}{48 \pi^{2} f^{2}}$,

where $m_{0}$ is the mass of the flavour- singlet field in the quenched effective lagrangian. For the chiral cutoff $\Lambda_{\chi}$, we chose $\Lambda_{\chi}=1 \mathrm{GeV}$. In fig. 1 we plot $m_{\pi}^{2} / m_{q}$ as a function of the quark mass, together with a fit to eq. (4), which yields $\delta=0.18(2)$, corresponding to $m_{0}=845(45) \mathrm{MeV}$.

Both for the nucleon and for the vector meson, quenched chiral perturbation theory predicts

$m_{H}=c_{H, 0}+c_{H, 1} m_{\pi}+c_{H, 2} m_{\pi}^{2}+\ldots$,

with $H \in\{\mathrm{N}, \rho\}$.

The coefficients $c_{N, 1}$ and $c_{\rho, 1}$ which are nonanalytic in the quark mass are special to the quenched approximation. In figs. 2 and 3 , we plot our data at $\beta=8.45$ together with fits to eq. (7).

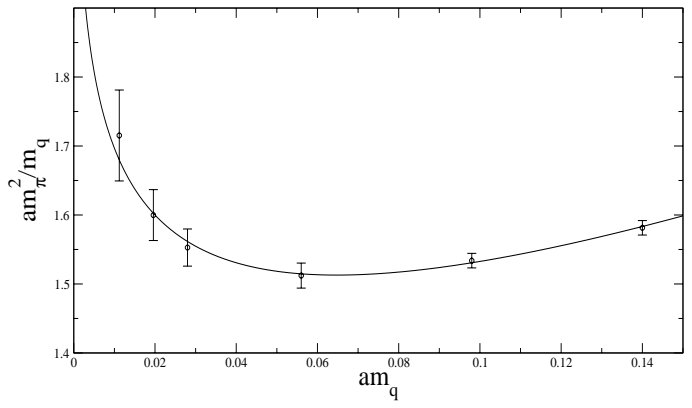

Figure 1. The ratio $m_{\pi}^{2} / m_{q}$, together with a fit to eq. (4) (at $\beta=8.45)$.

In order to investigate the scaling behaviour of our action, we plot data for the three lattice we have together in figs. 4 and 5 . For $m_{\pi}<500 \mathrm{MeV}$, discretisation errors appear to be very small.

\section{NUCLEON STRUCTURE}

Information about the internal structure of the nucleon is encoded in its structure functions. While they cannot be computed directly on the lattice, the operator product expansion (OPE) provides a connection between their moments and nucleon matrix elements of local operators. For instance, for the unpolarised structure function $F_{1}$, the OPE reads

$$
\begin{aligned}
& 2 \int_{0}^{1} d x x^{n-1} F_{1}\left(x, Q^{2}\right) \\
& =\sum_{f} E_{F_{1}, n}^{(f)} v_{n}^{(f)}+O\left(1 / Q^{2}\right),
\end{aligned}
$$

where $f$ denotes the quark flavour, $E_{F_{1}, n}^{(f)}$ is the (perturbative) Wilson coefficient and the matrix element $v_{n}^{(f)}$ is defined by

$$
\begin{aligned}
& \langle N(\vec{p})| O_{(f)}^{\left\{\mu_{1} \ldots \mu_{n}\right\}}-\text { traces }|N(\vec{p})\rangle \\
& =2 v_{n}^{(f)}\left(p^{\mu_{1}} \ldots p^{\mu_{n}}-\text { traces }\right)
\end{aligned}
$$




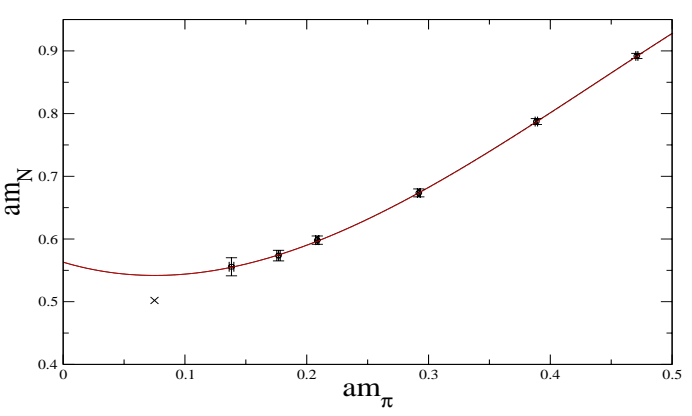

Figure 2. The nucleon mass, together with a fit to eq. (7).

with the operator

$O_{(f)}^{\mu_{1} \ldots \mu_{n}}=\bar{\psi}_{f} \gamma^{\mu_{1}}{\overleftrightarrow{D^{\mu_{2}}}}_{\mathrm{D}} \ldots{\stackrel{\leftrightarrow}{D_{n}}} \psi_{f}$

Similar relations hold for the other structure functions, see e.g. [1] for details. Both the matrix element $v_{n}^{(f)}$ and the Wilson coefficient $E_{F_{1}, n}^{(f)}$ depend upon the choice of a renormalisation scheme and scale; only in their product, these dependencies cancel.

The computation of matrix elements follows the procedure outlined in [1]: We form the ratio

$R=\frac{\left\langle N\left(t_{\text {sink }}\right) O(\tau) \bar{N}\left(t_{\text {source }}\right)\right\rangle}{\left\langle N\left(t_{\text {sink }}\right) \bar{N}\left(t_{\text {source }}\right)\right\rangle}$,

from which the matrix element can be extracted in the region $t_{\text {source }}<\tau<t_{\text {sink }}$. We always set $t_{\text {Source }}=0$ and $t_{\text {sink }}=13(9,7)$ on the $\beta=8.45$ $(8.0,7.6)$ configurations (in lattice units), which corresponds to a distance between source and sink of $1.4 \mathrm{fm}$.

The matrix elements we are considering are listed in table 4 , along with the operators used for their determination, where we use the operators (10) and

$O_{5,(f)}^{\mu_{1} \ldots \mu_{n}}=\bar{\psi}_{f} \gamma_{5} \gamma^{\mu_{1}} \overleftrightarrow{D}^{\mu_{2}} \ldots{\stackrel{\leftrightarrow}{D_{n}}}_{\nu_{f}}$

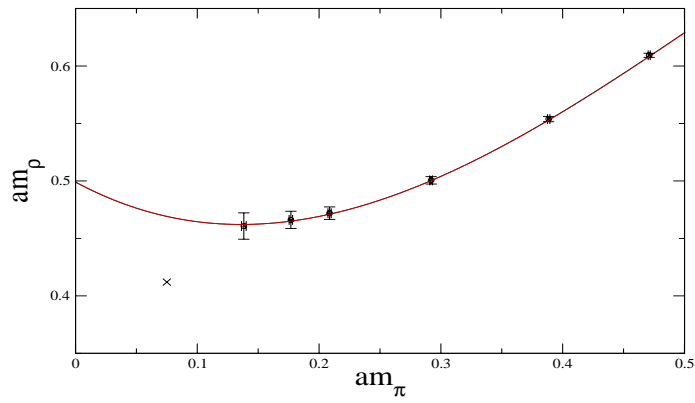

Figure 3. The vector meson mass, together with a fit to eq. (7).

We compute only flavour non-singlet matrix elements, since in this case there is no contribution from disconnected diagrams.

\section{NON-PERTURBATIVE MALISATION}

RENOR-

The operators appearing inside the three-point functions have to be renormalised. For $g_{A}=$ $\Delta u-\Delta d$, the operator to be used is the axial current $A_{\mu}$, the renormalisation of which is particularly simple because it does not depend on a renormalisation scheme or scale. It can be obtained from a Ward identity [6] as

$Z_{A}=\lim _{m_{q} \rightarrow 0} R_{Z_{A}}\left(m_{q}\right)$,

\begin{tabular}{|c|c|}
\hline Matrix Element & Operator \\
\hline$g_{A}$ & $\bar{\psi} \gamma^{5} \gamma^{2} \psi$ \\
$g_{T}$ & $\bar{\psi} \gamma^{5} \sigma^{24} \psi$ \\
$v_{2}$ & $O^{44}-\frac{1}{3}\left(O^{11}+O^{22}+O^{33}\right)$ \\
$a_{1}$ & $\frac{1}{2}\left(O_{5}^{24}+O_{5}^{42}\right)$ \\
\hline
\end{tabular}

Table 2

Operators used in the three-point functions. 


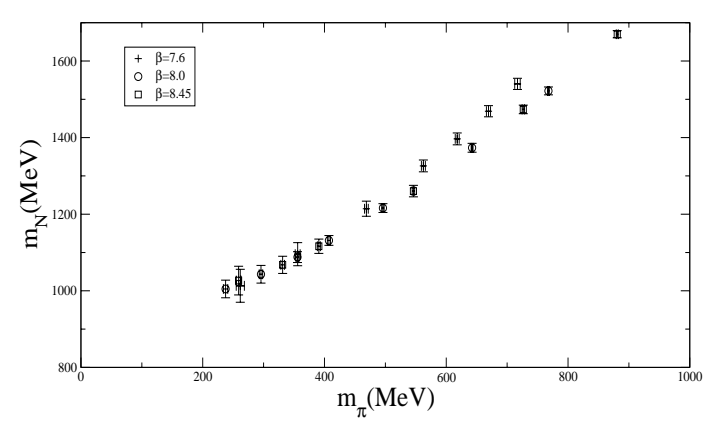

Figure 4. The nucleon mass for three different lattice spacings.

with

$R_{Z_{A}}\left(m_{q}\right)=\lim _{t \rightarrow \infty} \frac{2 m_{q}}{m_{\pi}} \frac{\langle P(t) P(0)\rangle}{\left\langle A_{4}(t) P(0)\right\rangle}$.

In fig. 6, we plot the ratio (14) as a function of the quark mass for the $\beta=8.45$ data set.

The renormalisation constants of the other operators under consideration are logarithmically divergent. We have computed them in the $\mathrm{RI}^{\prime}$ - MOM-scheme [7]. In this scheme, the renormalisation condition is formulated in terms of quark Greens functions in Landau gauge with an operator insertion at zero momentum transfer:

$C_{O}(p)=\frac{1}{V} \sum_{x, y, z} e^{-i p(x-y)}\langle\psi(x) O(z) \bar{\psi}(y)\rangle$.

From this quantity, the amputated vertex function $\Gamma_{O}$ is formed:

$\Gamma_{O}(p)=S^{-1}(p) C_{O}(p) S^{-1}(p)$,

with the quark propagator

$S(p)=\frac{1}{V} \sum_{x, y} e^{-i p(x-y)}\langle\psi(x) \bar{\psi}(y)\rangle$.

The renormalisation condition at scale $\mu$ is

$$
\left.Z_{\psi}(\mu) Z_{O}(\mu) \Pi_{O}\left(\Gamma_{O}(p)\right)\right|_{p^{2}=\mu^{2}}=1
$$



Figure 5. The vector meson mass for three different lattice spacings.

with the projector

$\Pi_{O}(\Gamma)=\frac{1}{12} \operatorname{tr}\left(\Gamma_{O, \text { Born }}^{-1}(p) \Gamma\right)$.

The wavefunction renormalisation constant $Z_{\psi}$ has been determined from the relation

$Z_{\psi} Z_{A} \Pi_{A}\left(\Gamma_{A}\right)=1$.

In order to convert the results to the $\overline{\mathrm{MS}}$ scheme, we first determine the renormalisation group invariant renormalisation constant $Z_{O}^{\mathrm{RGI}}$ :

$Z_{O}^{\mathrm{RGI}}=\left(Z_{O}^{\mathrm{RI}^{\prime}-\mathrm{MOM}, \mathrm{RGI}}(\mu)\right)^{-1} Z_{O}^{\mathrm{RI}^{\prime}-\operatorname{MOM}}(\mu)$,

and then convert to the $\overline{\mathrm{MS}}$-scheme at scale $\mu^{\prime}$ (we always use $\mu^{\prime}=2 \mathrm{GeV}$ ):

$Z_{O}^{\overline{\mathrm{MS}}}\left(\mu^{\prime}\right)=Z_{O}^{\overline{\mathrm{MS}}, \mathrm{RGI}}(\mu) Z_{O}^{\mathrm{RGI}}$,

with the conversion functions

$$
\begin{aligned}
& Z_{O}^{\mathcal{S}, \mathrm{RGI}}(\mu)=\left(2 b_{1} g^{\mathcal{S}}(\mu)^{2}\right)^{-\frac{d_{O, 1}}{2 b_{1}}} \\
& \cdot \exp \left[\int_{0}^{g^{\mathcal{S}}(\mu)} d \xi\left(\frac{\gamma_{O}^{S}(\xi)}{\beta^{S}(\xi)}+\frac{d_{O, 1}}{b_{1} \xi}\right)\right] .
\end{aligned}
$$

The coefficients of the $\beta$ and $\gamma$ functions are taken from $[8,9]$. 


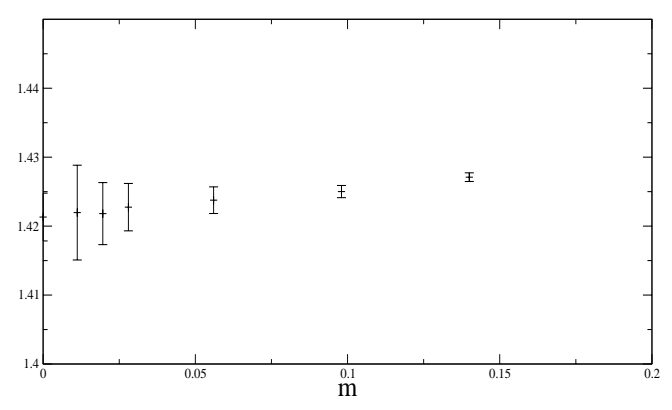

Figure 6. The ration $R_{Z_{A}}$ (eq. (14)), from which we obtain the renormalisation constant $Z_{A}$.

In fig. 7 we plot $Z_{v_{2 b}}^{\mathrm{RI}-\mathrm{MOM}}$ and $Z_{v_{2 b}}^{\mathrm{RGI}}$ for $\beta=8.45$. From the latter plot, we read off $Z_{v_{2 b}}^{\text {RGI }}=2.6$ from the plateau region $8 \mathrm{GeV}^{2}<$ $p^{2}<15 \mathrm{GeV}^{2}$. Using $Z_{v_{2 b}}^{\overline{\mathrm{MS}}, \mathrm{RGI}}(2 \mathrm{GeV})=0.737$, we obtain $Z_{v_{2 b}}^{\overline{\mathrm{MS}}}=1.92$. The renormalisation constants for all operators we need are shown in table 5. A comparison with results obtained in oneloop tadpole-improved lattice perturbation theory $[10,11]$ shows large discrepancies, especially for the operators with one derivative.

\section{RESULTS}

In table 6 we present values for the matrix elements which have been obtained from a linear extrapolation to the chiral limit.

Our results for the axial charge $g_{A}$ are displayed in fig. 8; Fig. 9 shows the tensor charge $g_{T}=$ $\delta u-\delta d$.

The results for the matrix elements $v_{2}^{u-d}=$ $\langle x\rangle^{u-d}$ and $a_{1}^{u-d}=2\langle x\rangle^{\Delta u-\Delta d}$ are shown in figs. 10 and 11, together with the phenomenological values. In both cases, there is almost no dependence on the quark mass visible. In the range $m_{\pi} \gtrsim 400 \mathrm{MeV}$, our results agree with previous results obtained from improved Wilson fermions [1].

\section{CONCLUSIONS}

The hadron mass dependence on the quark mass follows the predictions from quenched chiral perturbation theory. Furthermore, we find that discretisation errors are very small.

We have determined the flavour non-singlet nucleon matrix elements $g_{A}, g_{T}, v_{2}$ and $a_{1}$ doing the renormalisation nonperturbatively. Comparing the results at the three values for the lattice spacing we have, we find significant discretisation effects, in contrast with the situation for hadron masses.

While our results are in good agreement with earlier determinations, there remains a rather large discrepancy to the phenomenological values for $v_{2}$ and $a_{1}$, even at the lowest quark masses we can reach at present.

\section{ACKNOWLEDGEMENTS}

The numerical calculations were performed at the HLRN (IBM pSeries 690), at NIC Jülich (IBM pSeries 690) and at the PC farms at DESY Zeuthen and LRZ Munich. We thank these institutions for their support. Part of this work is supported by DFG under contract FOR 465 (Forschergruppe Gitter-HadronenPhänomenologie)

\section{REFERENCES}

1. M. Göckeler, R. Horsley, E.-M. Ilgenfritz, P. Rakow, G. Schierholz, A. Schiller, Phys. Rev. D53 (1996) 2317.

2. L. Giusti, M. Lüscher, P. Weisz, H. Wittig, Comp. Phys. Comm. 153 (2003) 31.

3. M. Lüscher, P. Weisz, Comm. Math. Phys. 97 (1985) 59.

4. C. Gattringer, R. Hoffmann, S. Schaefer, Phys. Rev. D65 (2002) 094503.

5. S. Capitani, M. Göckeler, R. Horsley, P.E.L. Rakow, H. Perlt, G. Schierholz, A. Schiller, Phys. Lett. B468 (1999) 150.

6. L. Giusti, C. Hoelbling, C. Rebbi, Nucl. Phys. Proc. Suppl. 106 (2002) 739. 



Figure 7. Left: The renormalisation constant $Z_{v_{2 b}}^{\mathrm{RI}-\operatorname{MOM}}(\mu)$, Right: The same, but with the renormalisation group running removed according to (21).

\section{Table 3}

\begin{tabular}{|c|c|c|c|c|c|c|}
\hline \multirow{2}{*}{ Operator } & \multicolumn{2}{|c|}{$\beta=7.6$} & \multicolumn{2}{c|}{$\beta=8.0$} & \multicolumn{2}{c|}{$\beta=8.45$} \\
\cline { 2 - 7 } & pert. & nonp. & pert. & nonp. & pert. & nonp. \\
\hline$O_{A_{\mu}}$ & 2.23 & $\mathrm{~b}$ & 1.36 & 1.59 & 1.30 & 1.42 \\
$O_{g_{T}}$ & 2.36 & $\mathrm{~b}$ & 1.36 & 1.73 & 1.33 & 1.54 \\
$O_{v_{2}}$ & 2.65 & $\mathrm{~b}$ & 1.33 & 2.11 & 1.39 & 1.92 \\
$O_{a_{1}}$ & 2.95 & $\mathrm{~b}$ & 1.34 & 2.21 & 1.40 & 1.98 \\
\hline
\end{tabular}

Comparison between the renormalisation constants $Z_{O}^{\overline{\mathrm{MS}}}(2 \mathrm{GeV})$ obtained non-perturbatively and in lattice perturbation theory.

7. G. Martinelli, C. Pittori, C. T. Sachrajda, M. Testa and A. Vladikas, Nucl. Phys. B $\mathbf{4 4 5}$ (1995) 81.

8. J. A. Gracey, Nucl. Phys. B 662 (2003) 247.

9. J. A. Gracey, Nucl. Phys. B 667 (2003) 242.

10. R. Horsley, H. Perlt, P. E. L. Rakow, G. Schierholz and A. Schiller [QCDSF Collaboration], Nucl. Phys. B 693 (2004) 3 [Erratum-ibid. B 713 (2005) 601].

11. R. Horsley, H. Perlt, P. E. L. Rakow, G. Schierholz and A. Schiller [QCDSF Collaboration], arXiv:hep-lat/0505015. 




Figure 8. The nucleon's axial charge as a function of the squared pion mass.



Figure 9. The nucleon's tensor charge as a function of the squared pion mass $(\overline{\mathrm{MS}}, 2 \mathrm{GeV})$.

Table 4

\begin{tabular}{|c|c|c|c|c|}
\hline$\beta$ & $g_{A}$ & $g_{T}$ & $v_{2}$ & $a_{1}$ \\
\hline 7.6 & $1.21(7)$ & $1.33(8)$ & $0.23(3)$ & $0.56(7)$ \\
8.0 & $1.37(5)$ & $1.35(4)$ & $0.28(2)$ & $0.58(4)$ \\
8.45 & $1.14(5)$ & $1.18(5)$ & $0.26(2)$ & $0.62(4)$ \\
\hline
\end{tabular}

Results for nucleon matrix elements in the chiral limit. 


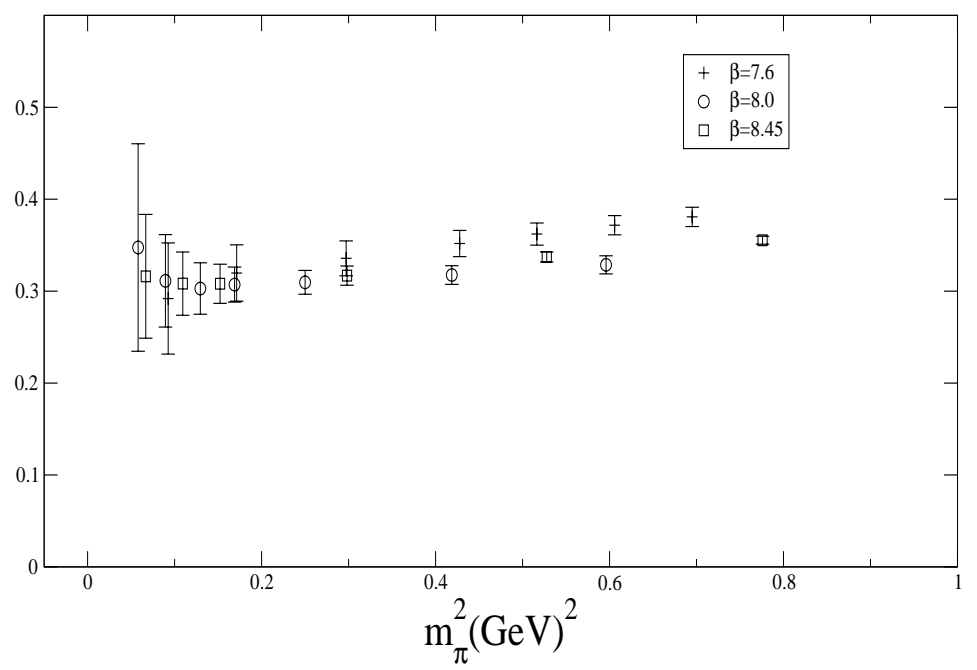

Figure 10. The nucleon matrix element $a_{1}^{u-d}$ as a function of the squared pion mass $(\overline{\mathrm{MS}}, 2 \mathrm{GeV})$.

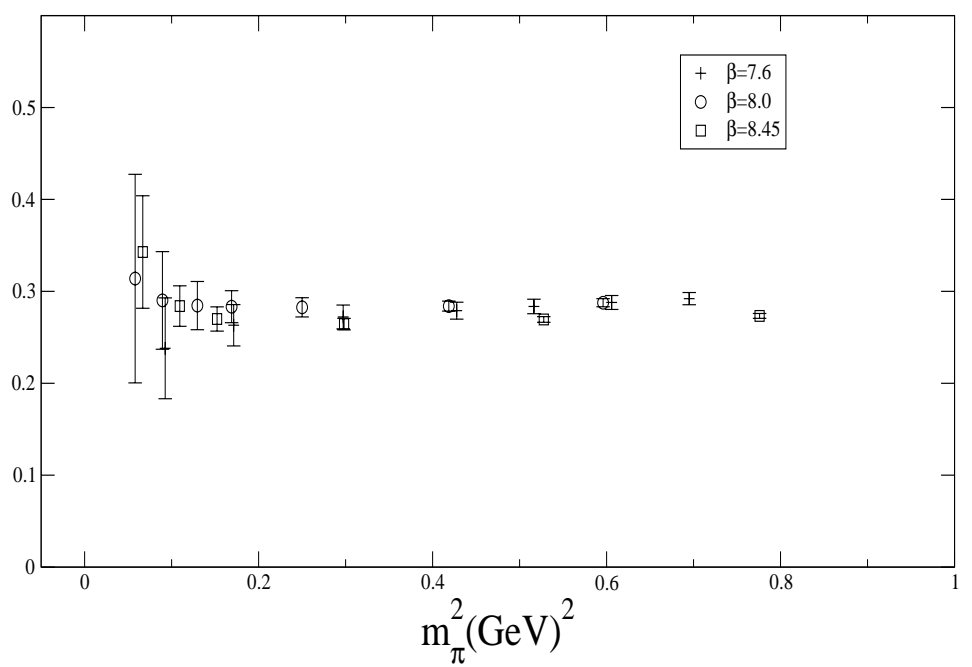

Figure 11. The nucleon matrix element $v_{2}^{u-d}$ as a function of the squared pion mass $(\overline{\mathrm{MS}}, 2 \mathrm{GeV})$. 